\section{Activation of the TRPM8 channel can generate analgesia in chronic neuropathic pain}

Peripheral or central activation of the TRPM8 (transient receptor potential melastatine family member 8) cool-sensitive ion channel can produce an analgesic effect, reversing hypersensitivity and reducing chronic neuropathic pain resulting from peripheral nerve damage, reports a paper in Current Biology. These findings are important given the dearth of treatments currently available for this severe clinical problem.

The TRPM8 channel is expressed by subsets of sensory neurons in dorsal root and trigenimal ganglia and is activated by cold or ligands, such as menthol or icilin, that trigger cold sensation. In a rat model of neuropathic pain, mild cooling of the skin or peripheral or central application of icilin produced marked analgesic effects, inhibiting sensitization of dorsal-horn neurons and facilitation of behavioral reflexes. Following nerve injury, TRPM8 expression was increased in a subset of sensory neurons. Further experiments confirmed TRPM8's importance in pain response and showed its analgesic effect to be centrally mediated and to rely on Group II/III metabotropic glutamate receptors (inhibitors of nociceptive responses). By contrast, results were obtained indicating that the TRPA1 coolsensitive ion channel is unlikely to have a role in analgesia.

The TRPM8 activators were needed at very low concentrations, produced few side effects, and also produced analgesic effects in sensitized pain states not induced by nerve injury. The authors, therefore, propose TRPM8 activators and downstream central mediators of TRPM8 action-such as Group II/III metabotropic glutamate receptors-as likely targets for the development of novel analgesics.

Original article Proudfoot CJ et al. (2006) Analgesia mediated by the TRPM8 cold receptor in chronic neuropathic pain. Curr Biol 16: 1591-1605

\section{Developmental abnormalities in the brains of patients with Prader-Willi syndrome}

Prader-Willi syndrome (PWS) is a complex neuroendocrine disorder, present from birth. Affected individuals are typically overweight, of short stature with low muscle tone, emotionally unstable, and have very mild to moderate learning disabilities. The demonstration in a quantitative analysis of brain development that patients with PWS have developmental abnormalities in specific areas of the brain provides a new insight into the pathophysiology of the syndrome.

The researchers use diffusion tensor imaging, based on high-field MRI, to examine the brains of eight patients with PWS and eight controls matched for age and gender. Higher trace value and reduced fractional anisotropy, both of which indicate deviation from regular development, were found in several specific brain regions in patients with PWS. Compared with controls, patients had a significantly higher trace value in the left frontal white matter and left dorsomedial thalamus (areas associated with psychiatric dysfunction; $P<0.05$ ). Additionally, patients had significantly reduced fractional anisotropy in the posterior limb of the internal capusle bilaterally (a region associated with motor dysfunction and perhaps connected to the 'central hypotonia' seen in Prader-Willi syndrome), and in the right frontal white matter and the splenium of the corpus callosum (areas relating to cognitive, visual, and spatial-perceptional function; $P<0.05$ for all). The affected regions identified in this study, therefore, correlate highly with the clinical features of PWS.

Original article Yamada K et al. (2006) Brain developmental abnormalities in Prader-Willi syndrome detected by diffusion tensor imaging. Pediatrics 118: e442-e448

\section{Atypical antipsychotics reduce prepulse inhibition deficits associated with schizophrenia}

Patients with schizophrenia are known to show impaired prepulse inhibition (PPI) of the startle reflex response, which correlates with their general inability to screen out irrelevant sensory input. A recent functional MRI study investigated the patterns of brain activation associated with PPI in schizophrenic patients, and assessed whether the type of antipsychotic medication administered affects this activation.

Kumari and co-workers recruited 12 healthy men, and 30 men with schizophrenia who were taking stable doses of a typical antipsychotic $(n=10)$ or of the atypical antipsychotics risperidone or olanzapine ( $n=10$ for both). The 